CDD: 501

\title{
TRUTH, LAWS AND THE PROGRESS OF SCIENCE MAURO DORATO
}

Department of Philosophy

University of Rome 3

Via Ostiense 234, 00144 Rome

ITALY

dorato@uniroma3.it

\begin{abstract}
In this paper I analyze the difficult question of the truth of mature scientific theories by tackling the problem of the truth of laws. After introducing the main philosophical positions in the field of scientific realism, I discuss and then counter the two main arguments against realism, namely the pessimistic metainduction and the abstract and idealized character of scientific laws. I conclude by defending the view that well-confirmed physical theories are true only relatively to certain values of the variables that appear in the laws.
\end{abstract}

Keywords: Truth. Laws. Progress. Commensurability.

\section{INTRODUCTION}

The question of the truth of well-confirmed scientific theories is one of the central questions of the philosophy of science, connected as it is to the ontology, the epistemology and the semantics of scientific theories. Such a question reduces, to a certain extent, to the question of the truth of the assertions expressing laws of nature, at least to the effect that the search for laws is considered to be the main goal of natural science, and of physics in particular.

In this paper I will defend the thesis that physical theories that have been greatly successful in the past (Newton's mechanics, to be specific) cannot be regarded as false in an absolute sense, or false tout court. Rather, Newton's mechanics and its laws are false or inaccurate (with respect to the phenomena it applies) only relatively to certain 
values of the magnitudes that appear in its fundamental laws. As a consequence, we could say that Newton's theory is not universally true - as Einstein's General Relativity (GR henceforth) could be if one had not to square it with quantum mechanics - but only relatively true, that is, true relatively to some values of the magnitudes that appear in its laws. This claim also entails that Newton's theory can be said to contradict currently accepted physical theories (special relativity, GR and quantum mechanics) only in a relative sense, since Newton's theory is a limiting case of quantum mechanics, special relativity and GR.

Consequently, scientific progress and continuity of science across major scientific revolutions is guaranteed by the generalization and progressive extension of the mathematical models used to represent physical phenomena. In order to express the thesis of continuity of science across major scientific revolutions in a clear way, I can do no better than quote Einstein:

Before the development of electrodynamics the laws of electrostatics were looked upon as the laws of electricity. At the present time we know that electric fields can be derived correctly from electrostatic considerations only for the case, which is never strictly realized, in which the electrical masses are quite at rest relatively to each other, and to the co-ordinate system. Should we be justified in saying that for this reason electrostatics is overthrown by the field-equations of Maxwell in electrodynamics? Not in the least. Electrostatics is contained in electrodynamics as a limiting case ; the laws of the latter lead directly to those of the former for the case in which the fields are invariable with regard to time. No fairer destiny could be allotted to any physical theory, than that it should of itself point out 
the way to the introduction of a more comprehensive theory, in which it lives on as a limiting case. (Einstein (1920, p. 102, italics added.))

The plan of the paper is as follows. In the next section I will present different ways of declining scientific realism, by distinguishing ontic, epistemic and semantic realism about scientific theories and laws. In section 3 I will discuss the two main arguments in favor of ontological and semantic anti-realism, namely the the so-called pessimistic metainduction and the abstract and idealized character of laws. In 4 and 5 I will briefly counter these two objections respectively. In 6 I will argue for a (moderate but robust enough) realism about laws of nature, and the view that continuity across major revolutions is ensured by the continuity of the mathematical structure entering into the definition of scientific models used by science.

\section{THE VARIOUS TYPES OF SCIENTIFIC REALISM}

Let us begin by endorsing Giere's view Giere (1990) about the relationship between theories, models and laws. Theories are to be regarded as a family of models, while models are to be regarded as abstract $n$-tuples whose essential features are defined by the scientific laws characterizing that theory. To be concrete, think of GR as constituted by a triple, namely by a manifold with a Lorentz signature $M$, a metric tensor $g$, and a tensor field $T$ specifying the matter-energy content: $\mathrm{GR}=\langle M, g, T\rangle$. Einstein's field equation (the main law of GR) defines the main features of the triple in question.

Finally, we need a theoretical hypothesis that specifies the relationship between the model and the world. The theoretical hypothesis is not a formal or physical hypothesis, but decidedly a philosophical hypothesis, advanced, however, on the basis of empirical and conceptual reasons. For instance, it could be the case that there is no relationship 
of representation or denotation between the model $\langle M, g, T\rangle$. and the physical world. Alternatively, it could be the case that the latter is partially or completely isomorphic to the former. It is in evaluating the theoretical hypothesis that the question of truth or realism of the laws or of the theories comes in.

Before giving more details, two terminological remarks are appropriate:

1. The realism in question in the expression 'scientific realism' is a word of art for referring to the unobservable world, where 'unobservable' means unobservable with our unaided senses.

2. In the expression 'ontic realism', realism is basically synonymous with a claim of independence of the unobservable world from subjects possessing minds, concepts and language.

We need to distinguish three different kinds of realisms about theories or laws, namely an ontological realism, an epistemic realism and a semantical realism.

Ontic realists maintain, consequently, that mature scientific theories and laws describe (and refer to) a mind-independent world, that is, a world inhabited by unobservable entities and/or structures that exist independently of us. Depending on whether ontic realists insists on the fact that science is about unobservable entities or unobservable structures or both, we may have different brands of ontic realism. Entity realists are ontic realist believing in the existence of unobservable objects endowed with at least some intrinsic property, where "intrinsic property" refers to properties that, like "having two ears", and unlike "being a brother", can be ascribed to an entity independently of the existence of other entities.

Ontic structural realists are ontic realists denying that, at the fundamental physical level, there exists entities, that is, objects endowed with intrinsic properties. On the contrary, according to the ontic struc- 
tural realists, science is about relational structures since the latter is all there is. Consequently, according to the ontic structural realist, "entities" ought to be reconceptualized as bundles of relations. Ontic structural realists typically deny the existence of entities because they hold that particles (fermions and bosons) are indiscernible and therefore (in virtue of Leibniz's Principle of the Identity of Indiscernible) cannot possess an identity and therefore be individuals. What both entity realists and ontic structural realists share is the claim that ontology and epistemology should be sharply separated: our epistemic resources are merely instruments to come to know a mind-independent world.

It won't be difficult to imagine that ontic antirealists deny the existence of, or suspend the judgment about, both entities and structures that are postulated to exist beyond the realm of appearances, and hold that we ought to believe just in laws or theories that describe, or refer just to, the level of directly observable phenomena (recall remark 1 above).

Epistemic realists about theories or laws claim, optimistically, that we are in the epistemic conditions of coming to know the regularities holding between unobservable entities, while epistemic antirealists, skeptically, hold that our epistemic powers are not sufficient to come to know the regularities holding in the unobservable world.

Finally, semantic realists about theories or laws hold that law statements are susceptible to being true or false, while semantical antirealist hold that theories or law statements are neither true nor false, but are devoid of a definite truth-value. Among the semantic realists I must also list the so-called "theory realists", who not only believe that scientific theories and laws have a definite truth-value, but that are in fact true, or approximately true.

The logical relationships between these three types of realism are not always easy to spell out. Due to lack of space, here I will simply 
note that semantic and ontic antirealism are typically motivated by epistemic antirealism, while semantic anti-realists about laws need not also be ontic anti-realists. Hacking (1983) and Cartwright (1983), for instance, have defended entity realism, but have denied that theories or laws can be true of the world, and count themselves as theory antirealists. Later we will briefly comment on the cogency of this move on the part of these two authors.

\section{THE TWO MAIN ARGUMENTS AGAINST ONTIC AND SEMANTIC REALISM}

The main arguments in favor of anti-realist stances about scientific theories and laws (in all three senses of realism) come from the history of science, and namely from a certain interpretation of two undeniable facts:

1. Science has an history;

2. Such a history is characterized by radical conceptual and methodological changes (scientific revolutions).

The basic question that needs to be raised at this point is: are such changes "cumulative" and "continuous" or rather "non-cumulative" and discontinuous? And what do cumulativity or discontinuity mean in this context? Since Kuhn [5], it is customary to identify non-cumulativity and discontinuity with the view that theory-change implies some sort of incommensurability between successive theories. Such an incommensurability involves not just concepts, aims and methods adopted before and after the scientific change, but also, crucially, the reference of certain key theoretical terms entering the laws of the pre-revolutionary and post-revolutionary theories. The latter historical claim generates the so-called "pessimistic meta-induction" [6], possibly the most powerful of the various anti-realist arguments about scientific laws and theories. A 
second important argument against scientific realism is also connected to questions of ontology and semantics, as it involves the idealization and abstraction typical of models and laws. Let me present these two objections in some more detail.

The first objection (the pessimistic meta-induction) arises because many central "theoretical terms" of past theories ("crystallin sphere", "caloric", "phlogiston", "ether", etc.), that were thought to refer to something real, in successive theories have been abandoned, since they have been discovered to be non-referential. Their ontological status today is not unlike that of the gods of the Greek mythology. Since the method used to postulate such terms is the same as that used today, we should conclude that also the current scientific theories ought to be regarded as false: by using induction on the destiny of past theories, also many of the terms of current and future theories will be discovered to be non-referential.

Notice that it is cold comfort to observe that in other historical cases other theoretical terms were not utterly abandoned, but changed completely their sense and therefore their reference. Think, for instance, of the use of "planet" in Ptolemy's and Copernicus' astronomy. In the former's framework, the term "planet" was used to refer to any heavenly body in motion, and therefore included the Sun, but excluded the Earth, conceived to be at rest. In the latter's framework, "planet" referred to bodies orbiting the Sun and included the Earth. The same holds for terms like space, time or momentum before and after Einstein's special relativity: the fact that momentum becomes dependent on the mass of the bodies should convince us of the fact that the meaning of terms of this kind holistically depends on the whole changing theoretical framework.

The second anti-realist objection has to do with the idealization and abstraction typical of scientific laws. For instance, the laws of inertia holds in the absence of forces or friction (abstraction from a real sit- 
uation), the simple pendulum law holds for inextensible or non-elastic threads (idealization), the Boyle law holds for molecules that do not attract each other (abstraction from real forces), and so on. How can we seriously maintain that laws describe or represent something in the world?

The importance of these two anti-realist arguments involves directly the question of the truth and of the explanatory power of scientific theories. In fact, if we had to conclude in favor of a radical discontinuity between, say, Copernicus's and Ptolemy's theories, Newton's and Einstein's, Newton's and Schrödinger's, we could not claim any sort of truth for science. And to the extent that laws are central to explain natural phenomena, but cannot be regarded as true, we could not legitimately claim that science explains natural phenomena.

In sum, the question of the cumulativity and continuity of scientific theories and laws across change does not involve the technological growth accompanying science, which is unquestionable, but rather the possibility of arguing that the history of science exemplifies cognitive growth toward theories coming closer to the truth, or toward more truthlike theories. Given that the notion of progress presupposes (in this case) a cognitive aim, the real question that is before is the following: can we claim that science progresses toward truer and more complete theories?

If we took seriously an analogy with biological evolution, we would have to conclude in favor of a negative answer, since we know that in the latter there was (and there is) no immanent, intrinsic goal toward which the evolution of species was directed. However, how seriously should we take the unquestionably important analogies between the evolution of science and the evolution of biological species? After all, scientists do have explicit goals (finding theories that are more and more experimentally accurate, for instance), goals that are taught from one generation to the next, and there is no analogy with the evolution of 
biological species in this aspect, given that the latter does not exemplify any consciously conceived goal (no intelligent design).

\section{COUNTERING THE PESSIMISTIC META-INDUCTION}

The arguments presented in this section and the next cannot be regarded as complete or thorough refutations of the two objections above. Nevertheless, they should be deemed sufficient to prepare the ground for the realistic position illustrated in the last section.

There are two ways to minimize the impact of the pessimistic metainduction:

1. Renouncing the claim that science is about entities, and follow the structural realists in arguing that science is apparently about entities, but is really only about structures.

2. Sticking with the view that science is about entities, but come up with a criterion on the basis of which one can separate the "good", trustworthy entities (that survive scientific change) from the bad, untrustworthy ones (that are thrown in the dust bin of history after scientific change).

1. According to the first counter-objection to the pessimistic metainduction - the one favored by the contemporary followers of Poincaré, Eddington and Cassirer (the so-called structural realists) - science comes closer to the truth when it is seen as describing the relations rather than the relata. The latter could either exist but be regarded as unknowable (as held by epistemic structural realist that thereby reintroduce in some sense Kant's unknowable noumena), or could just be regarded as non-existent (as is case with the already presented ontic structural realist, according to whom structure is all there is).

Leaving these rather academic distinctions, the important point is that structural realism was put forward in order to guarantee some 
continuity across scientific change, without having to risk the postulation of dubious entities Worrall (1989). The idea is that while relata can come and go, structures of physical relations, represented by our mathematical models, are stable across scientific revolutions and are therefore here to stay, because scientific change preserves the mathematical structures presupposed by the equations by generalizing and extending the structures:

"while real objects will always be hidden from our eyes.. the true relations between these objects are the only reality we can attain." (Poincaré (1905, p. 162.))

As Poincaré and Worrall after him have noted by using a brilliant case study, Fresnel's model of the optical ether was discarded in its ontological presupposition (the ether), but his equations were retained by Maxwell (and, one could add, further down the stream of history of physics till our days, to the extent that classical electromagnetism is a limiting case of quantum electrodynamics).

2. The second counter-objection bets on the existence of entities endowed with intrinsic properties, and therefore gives up structural realism. In so doing, it operates a distinction between detection properties and auxiliary properties of entities (Chakravartty (2007, p. 64)). The former properties, as advocated by Chakravartty, are "the causal properties one knows on the basis of detection" (Chakravartty (2007, p. 64)). These are properties that are responsible for the detection of certain entities in experimental settings. For instance, when one measures the mass/charge ratio of the electron, one performs certain experiments that enable one to assign certain causal roles to certain properties of electrons. Despite the fact that our theories on the electrons have changed in the course of the 20th century, the successful measurement of the charge or of the mass of these particles - via some causal mechanism used in the relevant experiments - is sufficient to claim that the 
relevant detection properties belong to an entity, and that the entity exists in virtue of the fact that it possesses those measured properties which cohere together. Furthermore, these properties do survive the change in whatever other properties are later assigned to the particle (for instance, the undulatory properties of self-interference, or spin etc. were discovered after the measurement of mass and charge).

How is this survival possible? The reader may remember that the causal theory of reference devised by Putnam was a way of rendering the existence of an entity wholly independent of the particular descriptions used to refer to it. According to Putnam, it is the causal contact with the entity which, like in a baptism, fixes the reference of the entity in question Putnam (1975). Analogously, according to Chakravartty, the detection properties result from our causal contact with the world, and from "a minimal interpretation of the equations of a theory". (Chakravartty (2007 p.51)). Here Chakravartty uses the typical arguments of the entity realists (Hacking (1983) and Cartwright (1983)): if you can interact indirectly with something in order to obtain another goal, say, charge a target negatively by using electrons, then you should assume that that something (your detection instruments, the electrons) exists.

The auxiliary properties, on the contrary, "cannot be determined on the basis of our causal contact with the world" (Chakravartty, ibid.), since they are either less directly connected, or are simply unconnected to our experimental practices. The idea is therefore that while detection properties survive scientific change, auxiliary properties can be discarded without damage to the continuity and progress of science. Given the vagueness of "less directly connected", however, this distinction may suffer from that post-hoc rationalization that Chakravartty has imputed to Stathis Psillos (1999 p.46). The latter scholar, for instance, has studied the caloric theory of heat (which erroneously presupposed the unexisting fluid called caloric) and the optical ether theo- 
ries (relying on the existence of the imaginary elastic solid called ether) and claimed that these were merely heuristic aids rather than essential components of the theory Psillos (1999).

However, it could be objected to both Psillos and Chakravartty that it is only in retrospect, and with the help of later theories, that one can demarcate detection (or essential) from auxiliary (or heuristic) properties. After all, how can we be sure about which properties or entities will be preserved and which not if we don't look at the course of history as our main epistemic criterion of demarcation?

The response to this objection is complicated, and here it can just be sketched by briefly mentioning an historical case. Take phlogiston, a chemical substance which, before Lavoisier, was thought to be involved in combustion and respiration in the following way: whenever something burns or rusts or breaths, it is because it releases an odorless, and colorless substance called phlogiston! After Lavoisier, oxygen theory explains all these processes by the claim that oxygen gets absorbed by burning objects and breathing animals: just the opposite process of phlogiston release.

First of all, we cannot claim that direct observations helps us to determine the truth of one of the two theories, since both phlogiston and oxygen are two theoretical terms, referring to substances that are both undetectable with the naked eye, and whose role in the observation can be reinterpreted according to the preferred theory:

when Priestley and Lavoisier watched the same experiment, Lavoisier should have seen what accorded with his theory that combustion and respiration are oxidation processes, while Priestley's visual experiences should have agreed with his theory that burning and respiration are processes of phlogiston release. Bogen (2009) 
Secondly, even supposing that we could refer to an existing entity (oxygen) by using a approximately correct description involving a fictional term (phlogiston), it would not be reasonable to claim that oxygen can be referred to as "dephlogisticated air". The idea of this description would arise from the fact that, say, in combustion, the nonexisting phlogiston is supposed to be released, while oxygen is something that in the same process gets absorbed. If we claim that during combustion air is deprived of phlogiston ("dephlogisticated"), we are somehow trying to capture the correct idea that combustion has to do with absorption rather than liberation of a given substance in air.

Despite the failure of these two moves, however, we can mention once again the essential fact noted by Hacking and Cartwright that measuring or indirectly manipulating an entity typically gives us sufficient reasons to believe in its existence (Dorato (1988)). Since the manipulation or measurement of properties of an entity involve by definition its detection properties, there seems to be a basis to distinguish properties that are more involved in our experimental interventions, and properties that are not so directly involved. The former call into question historically stable phenomenological laws, used by engineers and experimental physicists to interact with the entities in question. The latter involve either auxiliary, non-directly experimental parts of a theory, or simply more speculative laws that can be subject to radical change.

The distinction between two kinds of laws seems to be central to any attempt of claiming that one can be realist about entities and the relevant phenomenogical laws, while defending anti-realism about the more theoretical laws (Hacking (1983), Cartwright (1983)). However, the genuinity of this distinction can be questioned. Even the most theoretical law, if genuine and well-confirmed, should be able to indicate correlations between in principle observable magnitudes (think of Einstein's field equations, which, despite their theoretical character, are 
capable of indicating ways in which measurable quantities are correlated).

If it turned out that no distinction between phenomenological and theoretical laws is acceptable, a couple of important consequences would seem to follow. The first is that entity realism - our justified belief in the existence of unobservable entities - would entail not only a semantic realism about theories, but also what is called theory realism, namely the view that theories are true or approximately so. Otherwise, how could one effectively intervene in the unobservable world without relying on reliable theoretical descriptions of it?

A second important consequence of the collapse of the distinction between the two kinds of laws could be that also any distinction between auxiliary and detection properties would be very difficult to ground. However, the former distinction (holding between laws) need not be equated with the latter distinction (holding between properties). Auxiliary properties are not necessarily properties that are relevant for theoretical laws, but only intervene in those parts of the theory that do not lead directly to measurements.

All in all, it is not clear that one can distinguish between to-bepreserved entities - carrying detection properties - and to-be-abandoned entities - carrying auxiliary properties - simply by looking at the structure of a theory at any given moment of time. The fact that, in order to be able to demarcate detection from auxiliary properties, the realist may need history, and therefore some amount of post-hoc rationalization, could not be so disastrous, after all. Maybe a recourse to a longer unit of time, of the type of Lakatos' research programs, is unavoidable, and some degree of retrospection has to be accepted in any realistic account of science. However, for the realist it is not so important not to be able to know, at any moment of time, which properties count as essential and which are auxiliary, as long as she can be sure that some of these properties will be able to survive also after a radical scientific 
change. Which properties will survive is of course a question that ought to be decided by experts (philosophers and scientists) only thanks to a case by case analysis, since I doubt that an algorithm exists that can apply to all possible theories and entities. And, in any case, continuity of mathematical structure - the way of structural realism - is always open for a realist defense of continuity and therefore for some kind of scientific realism.

\section{THE ANTIREALISM COMING FROM THE IDEALIZED FEATURES OF LAWS}

The second objection mentioned above has to do with the fact that a real pendulum, a body falling in a dense medium, a gas whose molecules exert non-negligible forces amongst each other, and a real liquid characterized by viscosity, do not literally obey the laws defining those simplified models in which the corresponding effects are neglected. The real behavior of the above physical systems at best approximates the idealized behavior described by models in which a pendulum and bodies move in a frictionless vacuum, molecules do not attract each other, and liquids are not viscous.

The first question we should raise is the following: are the idealization and abstraction mentioned in these models unavoidable, or can other less abstract or idealized models eliminate them? Naturally, it is possible to construct more complex models, in which one introduces either forces of friction that are proportional to the velocity of a body in a dense material (like the model for the motion of a parachute), or forces of attraction between molecules of a gas, together with their volume, as in van der Walls' law below (2). Such a law extends to real gases the domain of applicability of the law characterizing perfect gases (1), expressed in the form: 


$$
P V=K N T
$$

and renders its predictions more general and accurate:

$$
\left[P+a(N / V]^{2}(V-N b)=K N T\right.
$$

Apart from the substitution of the constant $K$ with the product of $N$ (the total number of gas molecules) $K$ (the so-called Boltzmann's constant) and the absolute temperature $T,(1)$ above is substantially Boyle's law. In (2), $P$ is the pressure of the gas, $V$ is the volume, and $a$ and $b$ are two experimentally determinable constants, characteristic of every single gas.

The fundamental point is that with respect to the behavior of a real gas, also van der Walls' law (2) derives from an abstraction and an idealization: the effect of the molecular forces for the $N$ molecules is actually of the order of $N(N-1)$, which can be approximated with $N^{2}$ only for very large $N$, as in the above equation. For how effectively large $N$ may be in a gas, the implicit idealization in the law is evident, given that not all molecules of a given gas have a volume exactly proportional to $b$.

A philosophical theory of the scientific laws that does not recognize that such an modeling aspect of science is unavoidable is bound to misrepresent the concrete functioning of science. Not only can any physical theory be appropriately identified with a class of abstract models in the sense of model theory - recall GR as a triple containing M, $\mathrm{g}$ and $\mathrm{T}$ - but in every scientific theory, the concept of the model in another, distinct sense, is always present. Such a second sense, given by a "simplified and schematic representation of phenomena", should not be confused with the first, but is as important as the first.

How serious is the threat of this sort of idealization to a realist understanding of laws and theories? 


\section{A MODERATE REALISM ABOUT LAWS AND SCIENCE}

An antirealistic viewpoint about laws has often been defended in the past. The first neopositivist philosophers regarded law-like generalizations as being purely instrumental for predictive purposes. In this empiricist tradition, laws are devoid of definite truth-values, as they are regarded as being only schemes for making meaningful predictions. It is not just that laws are to be treated as "inference tickets" from one set of measurements/observations to another; furthermore, if laws refer to an infinite domain of objects, they cannot be verified, since we cannot in principle observe an infinite number of cases. Moreover, how can we consider "symbolic generalizations" or "exemplars" (Thomas Kuhn's terms), like those of Newton's three laws, as statements about the world, before the specification of particular force functions? More recently, even if for reasons that are different from those given by the first neopositivists, contemporary philosophers like Giere have considered laws to be true only in an idealized way - or only true in the model.

This antirealistic conclusion, however, need not follow. Despite the fact that they are idealized and valid only in certain circumstances, laws are not mere conventions lacking in empirical content, given that they refer to the world of phenomena by approximation; in other words, laws reproduce the structures of experience through partial isomorphisms dictated by the particular mathematical models at hand (French, da Costa (2003), French et. al. (2002)). These isomorphisms originate both from the measurement of data Dorato (2005), and from the real properties of bodies as opportunely idealized by the models. A real pendulum "resembles" Galileo's, despite the fact that the mathematical model satisfying the law of isochronism abstracts from friction and from the weight of the string, idealizing its inextensibility. In other words, the model attributes the idealized pendulum (the abstract "object" that fully satisfies Galileo's law) a few characteristics that real 
pendulums do possess, but not to the idealized degree that is typical of the model. The same idea applies to the law of ideal gases, which idealizes the properties of real gases, but abstracts from intermolecular forces, while van der Waals's law does not abstract from such force, but idealizes and simplifies them. It is clear therefore that, due to the abstraction and idealization of any scientific model, in well-defined experimental contexts such models can be applied only within a certain degree of approximation. It seems to follow that the notion of truth for laws should be correspondingly be valid only "with some approximation".

However, here comes the rub. The attribution of a degree to truth, and a talk of verisimilitude or truth-likelihood, forces us to introduce a metric needed to measure the distance of the currently accepted theory from the true theory, which, however, we typically ignore. In addition, there is the further difficulty, that can certainly be circumvented, that the meaning of the very notion of truth, at least intuitively, does not allow for degrees - a sentence is either true or false. Given these difficulties, shouldn't one conclude that laws, qua definitions of the models in which they intervene, are, like the latter, neither true nor false, but simply more or less adequate to the descriptive purpose, or are more or less effective in their predictive duties, etc.? After all, one map is not more or less true than another.

This conclusion need not follow. In virtue of the approximate degree to which some properties of real pendulums are indeed reflected by their abstract model, the notion of truth that is applicable in this case is suggested by the partial isomorphisms linking the data models and the theoretical models in which laws hold with truth. Here I can only direct the reader to some important contributions, and to the literature therein indicated (French, da Costa (2003), French et. al. (2002)).

To sum up, there is a further sense of approximation of laws which is not just given by their abstracted character, but by the fact that sci- 
ence progresses via generalization of structures. In effect, if we wished to discuss the truth of the laws, we should in any case discuss it both in terms of truth relative to their domains of application, and in terms of the the limits of approximation permitted by the model. In any case, claiming that laws are valid within the limits of their domain of application and within the limits of approximation allowed by the model for example, the law of the simple pendulum is valid/true only for very small angles of oscillation, where the sine of the angle $x$ can be approximated by $x$ - is the same as claiming that the law in question, strictly speaking, is only true in the mathematical models that it contributes to defining.

But this fact has no antirealist implication. Newton's mechanics for example is "true" in its area of application, in the sense that it "works very well", or gives reliable descriptions of what happens for bodies moving at speeds that are slow with respect to the speed of light (where effects described by special relativity are negligible), for so called-classical bodies whose dimensions are far superior to those of atoms (where typical quantum effects are usually not present), and for bodies in which gravitational fields are weak (so that GR is not necessary). From this point of view, and contrary to Popper's falsificationism, science should not therefore be seen as a sequence of "definitive abandonment" of theories and scientific laws which were previously held as valid. Good theories will never turn into useless, dead weights to be rid of; Newton's mechanics is still indispensable for sending satellites into orbit.

Once we have understood that any nomic generalization is not universal because laws can never be applied to all values of the physical parameters which intervene in them, the growth of scientific knowledge, or scientific progress tout court can and must be seen as a progressive discovery of the limits of application of scientific laws. Like the process of personal maturity, a greater knowledge of one's self involves, in a 
certain sense, an awareness of one's own limits.

Another important philosophical consequence of this viewpoint of scientific laws should be highlighted: if a law of physics is essentially valid for only a few values of parameters, and not for all possible values, it is difficult to imagine that one day we may discover a single universal law which can summarize - and from which we may deduce - all of our knowledge of nature, human beings included. From this point of view, it is therefore legitimate to salvage one important element of Popper's view of science, that on the basis of which the scientific enterprise is "an endless quest."

\section{REFERENCES}

BOGEN, J. "Theory and Observation in Science", in E. Zalta (ed.), Stanford Encyclopedia of Philosophy URL:http://plato.stanford. edu/entries/science-theory-observation/, 2009.

CARTWRIGHT, N. How the laws of physics lie, Cambridge University Press, Cambridge, 1983.

CHAKRAVARTTY, A. A Metaphysics for Scientific Realism, Cambridge University Press, Cambridge, 2007.

DORATO, M. "The world of the worms and the quest for Reality", Dialectica, 42, p.171-182, 1988.

- The Software of the Universe, Ashgate, 2005.

FRENCH S. and DA COSTA, N. Science and Partial Truth: A Unitary Approach to Models and Reasoning in Science, Oxford University Press, New York, 2003.

FRENCH, S. BUENO O. and LADYMAN J. "On Representing the Relationship between the Mathematical and the Empirical", Philosophy of Science 69, p. 452-73, 2002. 
EINSTEIN, A. Relativity: The special and general theory. A popular exposition, transl. by Robert W. Lawson, Methuen and Co Ltd, 1920.

GIERE, R. Explaining Science, Chicago University Press, Chicago, 1990.

HACKING, I. Representing and Intervening, Cambridge University Press, Cambridge, 1983.

KUHN, T. The Structure of Scientific Revolutions, Chicago University Press, Chicago, 1962.

LAUDAN, L. "A Confutation of Convergent Realism", Philosophy of Science, 48, 1, p. 19-49, 1981.

POINCARÉ H. La science et l'hypothèse. Paris: Flammarion, 1905.

PSILLOS, S. How Science tracks truth, Routledge, London, 1999.

PUTNAM, H. "The meaning of meaning", in Mind, Language and Reality, Cambridge University Press, Cambridge, vol II, p.215-271, 1975.

WORRALL, J. "Structural Realism: The Best of all Possible Worlds?" Dialectica 43, p. 99-124, 1989. 